\title{
Classification of Rational Homotopy Type for 8-Cohomological Dimension Elliptic Spaces
}

\author{
Mohamed Rachid Hilal ${ }^{1}$, Hassan Lamane ${ }^{1}$, My Ismail Mamouni ${ }^{2 *}$ \\ ${ }^{1}$ Faculté des Sciences Aïn Chock, Casablanca, Morocco \\ ${ }^{2}$ Centre Pédagogique Régional, Rabat, Morocco \\ Email: \{rhilali, hlamanee\}@hotmail.com, *mamouni.myismail@gmail.com
}

Received September 21, 2011; revised November 8, 2011; accepted November 15, 2011

\begin{abstract}
The different methods used to classify rational homotopy types of manifolds are in general fascinating and various (see $[1,7,8])$. In this paper we are interested to a particular case, that of simply connected elliptic spaces, denoted $X$, by discussing its cohomological dimension. Here we will the discuss the case when $\operatorname{dim} H^{*}(X ; \mathbb{Q})=8$ and $\chi(X)=0$.
\end{abstract}

Keywords: Rational Homotopy Theory; Elliptic Spaces; Classification; Rational Homotopy Type; Minimal Model of Sullivan

\section{Introduction}

Let us first recall some basic definitions of rational homotopy theory. A simply connected space $X$ is called elliptic, if both of $H^{*}(X ; \mathbb{Q})$ and $\pi_{*}(X) \otimes \mathbb{Q}$ are finite dimension, and that its cohomological Euler-Poincar characteristic is given as $\chi_{c}(X):=\sum_{k \geq 0}(-1)^{k} \operatorname{dim} H^{k}(X ; \mathbb{Q})$. We will fix this throughout this paper. The space is called rational if $\pi_{*}(X)$ is a $\mathbb{Q}$-vector space. If it is not, by [4], we can associate a rational simply connected space, denoted $X_{\mathbb{Q}}$, verifying

$$
\begin{array}{lll}
H^{*}\left(X_{\mathbb{Q}} ; \mathbb{Q}\right) & \mathbb{Q} H^{*}(X, \mathbb{Q}) & \text { asalgebras, } \\
\pi_{*}\left(X_{\mathbb{Q}}\right) & \mathbb{Q} \pi_{*}(X) \otimes \mathbb{Q} & \text { asvectorspaces. }
\end{array}
$$

The rational homotopy type of $X$ is defined as the homotopy type of its rationalization $X_{\mathbb{Q}}$. Our purpose in this paper to give a complete classification this rational homotopy type when $\operatorname{dim} H^{*}(X ; \mathbb{Q})$ and $\chi_{c}(X)=0$.

\section{Preliminaries}

The rational homotopy theory was founded in the the end of the sixties by Daniel Quillen and Denis Sullivan. One of the technical gadget of this theory is the minimal model of Sullivan, it is a free $\mathbb{Q}$-commutative differential graded algebra $(\Lambda V, d)$ associated to any simply connected CW complex $X$ of finite type [3]. Here $V=\oplus_{i \geq 2} V^{i}$ is $\mathbb{Q}$-graded vector space with $\operatorname{dim} V^{i}<\infty$ and $d$ a decomposable differential; that means $d V^{i} \subset\left(\Lambda^{\geq 2} V\right)^{i+1}(d$ does not have a linear part) and that

\footnotetext{
"Corresponding author.
}

$d^{2}=0$. It is well known that the minimal model $(\Lambda V, d)$ determines the rational homotopy type of $X$, in the sense that

$$
\begin{array}{lll}
H^{*}(X ; \mathbb{Q}) & \simeq H^{*}(\Lambda V, d) & \text { asalgebras } \\
\pi_{*}(X) \otimes \mathbb{Q} \simeq V & & \text { asvectorspaces. }
\end{array}
$$

For example, the minimal model of an even sphere $\mathbb{S}^{2 n}$ is of the form $(\Lambda\{x, y\}, d)$ with $|x|=2 n,|y|=4 n-1$, $d x=0, d y=x^{2}$ and $H^{*}\left(\mathbb{S}^{2 n} ; \mathbb{Q}\right) \cong \mathbb{Q}[x] / x^{2}$, while the minimal model of an odd sphere $\mathbb{S}^{2 n+1}$ is of the form $(\Lambda\{x, y\}, d)$ with $|y|=2 n+1, d y=0$. It will be utile for our proofs, to recall the reader this simple properties. For a homogeneous element $x$ of $\Lambda V,|x|$ denotes its degree, which verifies the following:

- $x y=(-1)^{|x||y|} y x$;

- $\quad d(x y)=(d x) y+(-1)^{|x|} x d y \quad$ (Leibniz formula).

In particular $x^{2}=0$, when $|x|$ is odd and $x y=y x$ when $|x|$ is even.

$\chi_{\pi}(X):=\sum_{k>0}(-1)^{k} \operatorname{dim} V^{k}$ is called the homotopic Euler-Poincar characteristic of $X$. In [5], S. Halperin have shown the following:

$$
\begin{array}{rlc}
\chi_{c} \geq 0 & \text { and } & \\
\chi_{c}>0 & \Leftrightarrow & \chi_{\pi}=0 \\
& \Leftrightarrow & H^{\text {odd }}(\Lambda V, d)=0
\end{array}
$$

One other notion that we will use throughout this paper is the formal dimension of $X$, given as $f d(X):=\max \left\{n, H^{n}(X ; \mathbb{Q}) \neq 0\right\}$. We know from [5] that, when $a_{1}, \cdots, a_{n}$ are the elements of an homogene- 
ous basis of $V$,

$$
f d(X)=\sum_{\left|a_{i}\right| \text { odd }}\left|a_{i}\right|-\sum_{\left|a_{i}\right| \text { even }}\left(\left|a_{i}\right|-1\right) .
$$

Our proofs are essentialy based on this equality combined with an other equality established by J. Friedlander and S. Halperin in [2], that

$$
\begin{aligned}
& \sum_{\left|a_{i}\right| \text { even }}\left|a_{i}\right| \leq f d(X) \\
& \sum_{\left|a_{i}\right| \text { odd }}\left|a_{i}\right| \leq 2 f d(X)-1 .
\end{aligned}
$$

Finally, let us recall that $H^{*}(X ; \mathbb{Q})$ satisfies the Poincar duality, that means that the multiplication $H^{k}(X ; \mathbb{Q}) \times H^{n-k}(X ; \mathbb{Q}) \rightarrow H^{n}(X ; \mathbb{Q}) \cong \mathbb{Q} \mu$ is a non degenerate bilinear form (here $n=f d(X)$ and $\mu$ denotes the so called fundamental class of $H^{*}(X ; \mathbb{Q})$ ). For the reader interested by more details about the rational homotopy theory, we recommend the basic reference [3].

\section{The Main Theorem}

In all the remainder of this paper, $X$ denotes a simply connected elliptic space with $\operatorname{dim} H^{*}(X ; \mathbb{Q})=8, \chi_{c}=0$ and $(\Lambda V, d)$ will denotes it minimal model. Put $\left\{1, \alpha_{1}, \cdots, \alpha_{6}, \mu\right\}$ a basis for $H^{*}(X ; \mathbb{Q})$ with the condition that $\left|\alpha_{i}\right| \leq\left|\alpha_{i+1}\right|$ and that $a_{i} \in \Lambda V$ with $\left[a_{i}\right]=\alpha_{i}$. The following table summarizes the classification of its rational homotopy type.

\begin{tabular}{ll}
\hline Rational homotopy type of $X$ & \multicolumn{1}{c}{ Legend } \\
\hline$\left(\mathbb{S}^{2 k+1}\right)^{3}$ & $f d(X)=3(2 k+1)$ \\
$\mathbb{S}^{n} \times \mathbb{S}^{n} \times \mathbb{S}^{2 n}$ & $f d(X)=4 n$ and $n$ is odd \\
$\left(\mathbb{S}^{n} \times \mathbb{S}^{n} \times \mathbb{S}^{2 n}\right) \# \mathbb{S}_{(2)}^{2 n}$ & $f d(X)=4 n$ and $n$ is odd \\
$\mathbb{S}^{2 k+1} \times \mathbb{S}^{2 k+1} \times \mathbb{S}^{2(k+p)}$ & $f d(X)=2(2 k+1)+2(k+p)$ \\
$\left.\mathbb{S}^{2 n} \bigotimes_{d_{2}} \mathbb{S}^{n} \times \mathbb{S}^{2 n}\right) \times \mathbb{S}^{2(n+p)+1}$ & $f d(X)=4 n+2(n+p)+1$ \\
$\mathbb{S}^{2 n} \times \mathbb{S}^{2 n} \times \mathbb{S}^{2(n+p)+1}$ & $f d(X)=4 n+2(n+p)+1$ \\
$\mathbb{S}^{2 n} \times\left(\mathbb{S}^{2(n+p)+1}\right)^{2}$ & $k \geq 2 n+2$ \\
$\mathbb{S}^{2 n} \times \mathbb{S}^{k} \times \mathbb{S}^{k}$ & $\lambda \in \mathbb{Q}^{*}$ \\
$\mathbb{S}^{2 n+1} \times Y_{\lambda}$ & \\
$\mathbb{S}^{2 n} \times \mathbb{S}^{2 k+1} \times \mathbb{S}^{2(n+k)+1}$ & \\
$\mathbb{S}^{2 n+1} \times \mathbb{S}^{2(n+k)+1} \bigotimes_{d} \mathbb{S}^{2 k}$ & \\
$\mathbb{S}_{(3)}^{n} \times \mathbb{S}^{2 k+1}$ & \\
$\mathbb{S}^{2 k_{1}+1} \times \mathbb{S}^{2 k_{2}+1} \times \mathbb{S}^{2 k_{3}+1}$ & \\
$E$ & \\
&
\end{tabular}

Legend: 1) In [6], I. M. James has introduced the concept of reduced product when $X$ is a based space. He put $X_{(1)}:=X$ and

$$
X_{(p)}:=X \times \cdots \times X /\left(x_{1}, \cdots,{ }^{*}, \cdots, x_{p-1}\right) \sim\left({ }^{*}, x_{1}, \cdots, x_{p-1}\right) .
$$

2) From this construction applied to an even sphere $\mathbb{S}^{n}$ arises the James sphere $\mathbb{S}_{(p)}^{n}$, satisfying $H^{*}\left(\mathbb{S}_{(p)}^{n} ; \mathbb{Q}\right) \simeq \mathbb{Q}[a] /\left(a^{p+1}\right)$. The use of the denotation $\mathbb{S}_{(p)}^{n}$ means implicitly that $n$ is supposed to be even.

As the most of our proofs will be by contradiction, we will mark such proofs by (by contradiction) in its beginning and by (QED) when its end. In the spirit and desire to simplify the lecture of this paper, we will subdivide it on many propositions, lemmas and theorems. The first one is that:

Lemma 1 There exists $i \in\{1, \cdots, 5\}$ such that $\left|\alpha_{i}\right|<$ $\left|\alpha_{i+1}\right|$.

Proof. Suppose that $\left|\alpha_{i}\right|=\left|\alpha_{i+1}\right|=n$, then $\chi_{c}=1+(-1)^{f d(X)}+5 \times(-1)^{n} \neq 0$.

\subsection{The Case Where}

$$
\left|\alpha_{1}\right|=\left|\alpha_{2}\right|=\left|\alpha_{3}\right|<\left|\alpha_{4}\right|=\left|\alpha_{5}\right|=\left|\alpha_{6}\right|
$$

Proposition 2 If $\left|\alpha_{1}\right|=\left|\alpha_{2}\right|=\left|\alpha_{3}\right|<\left|\alpha_{4}\right|=\left|\alpha_{5}\right|=\left|\alpha_{6}\right|$, then $X$ has the rational homotopy type (r.h.t) of $\left(\mathbb{S}^{2 k+1}\right)^{3}$, with $3(2 k+1)=f d(X)$ and $k \in \mathbb{N}^{*}$.

Proof. Since $\left|\alpha_{1}\right|=\left|\alpha_{2}\right|=\left|\alpha_{3}\right|$, then $a_{1}, a_{2}, a_{3} \in V$. We distinguish two cases:

1) $\left|\alpha_{1}\right|$ is odd. Then $\alpha_{1}^{2}=\alpha_{2}^{2}=\alpha_{3}^{2}=0$. Let $E$ be the vector space spanned by $\alpha_{1} \alpha_{2}, \alpha_{1} \alpha_{3}, \alpha_{2} \alpha_{3}$.

- If $\operatorname{dim} E=3$, we can take $\left\{\alpha_{1} \alpha_{2}, \alpha_{1} \alpha_{3}, \alpha_{2} \alpha_{3}\right\}$ as a basis of $E$. Let $\left\{b_{1}, \cdots, b_{n}\right\}$ an homogeneous basis of a complement of $\mathbb{Q}\left\{a_{1}, a_{2}, a_{3}\right\}$ in $V$ with $\left|b_{1}\right| \leq \cdots \leq\left|b_{n}\right|$ and $d b \in \Lambda^{\geq 2}\left\{a_{1}, a_{2}, a_{3}\right\}$, therefore $d b_{1}=0$ and $\Lambda\left\{\left[a_{1}\right],\left[a_{2}\right],\left[a_{3}\right]\right\} \oplus \mathbb{Q}\left[b_{1}\right] \subset H^{*}(\Lambda V, d)$, what implies that $\operatorname{dim} H^{*}(\Lambda V, d) \geq 9$. So the minimal model of $X$ is $\left(\Lambda\left\{a_{1}, a_{2}, a_{3}\right\}, d\right)$ with $d a_{i}=0$ and $\left|a_{i}\right|=2 k+1$. This is exactly the minimal model of $\left(\mathbb{S}^{2 k+1}\right)^{3}$.

- If $1 \leq \operatorname{dim} E \leq 2$, the there exist $\lambda_{1}, \lambda_{2} \in \mathbb{Q}$ such that $\lambda_{1} \alpha_{1} \alpha_{2}+\lambda_{2} \alpha_{1} \alpha 3+\alpha_{2} \alpha_{3}=0$, and then $d a_{4}=\lambda_{1} a_{1} a_{2}+\lambda_{2} a_{1} a 3+a_{2} a_{3}$. According to the Poincar duality, we have $\alpha_{1} \alpha_{2} \alpha_{3}=\mu$, so $d\left(a_{1} a_{4}\right)=$ $a_{1} a_{2} a_{3}$ and $\left[a_{1} a_{2} a_{3}\right]=\mu=0$. This is impossible.

2) $\left|\alpha_{1}\right|$ is even. Then $f d(X)$ and $\left|\alpha_{4}\right|$ are odd, because that $\chi_{c}=1+(-1)^{f d(X)}+3+(-1)^{\left|\alpha_{4}\right|}=0$. Therefore $\alpha_{1}^{2}=\alpha_{2}^{2}=\alpha_{3}^{2}=\alpha_{1} \alpha_{2}=\alpha_{1} \alpha_{3}=\alpha_{2} \alpha_{3}=0$, and there exist tree generators $b_{i}$ of $\Lambda V$ with even degrees such that $d b_{i}=a_{i}^{2}, \quad i=1 \cdots 3$. Then

$$
\sum_{\left|a_{i}\right| \text { even }}\left|a_{i}\right| \geq 3\left|a_{4}\right|+3\left|b_{1}\right| \geq 3\left|a_{4}\right|+6\left|a_{1}\right|-3>2 f d(X)-1 \text {. }
$$


This is impossible.

\subsection{The Case Where}

$$
\left|\alpha_{1}\right|=\left|\alpha_{2}\right|<\left|\alpha_{3}\right|=\left|\alpha_{4}\right|<\left|\alpha_{5}\right|=\left|\alpha_{6}\right|
$$

Lemma 3 If $\left|\alpha_{1}\right|=\left|\alpha_{2}\right|<\left|\alpha_{3}\right|=\left|\alpha_{4}\right|<\left|\alpha_{5}\right|=\left|\alpha_{6}\right|$, then $f d(X)$ and $\left|\alpha_{3}\right|$ are even, $\left|\alpha_{1}\right|$ is odd, and $\alpha_{1} \alpha_{2} \neq 0$.

Proof. First, because of the Poincar duality, we have $f d(X)=\left|\alpha_{1}\right|+\left|\alpha_{6}\right|=2\left|\alpha_{3}\right|$ is even, and $\left|\alpha_{1}\right|,\left|\alpha_{6}\right|$ have the same parity. Hence $0=\chi_{c}=2+4(-1)^{\left|\alpha_{1}\right|}+2(-1)^{\left|\alpha_{3}\right|}$, $\left|\alpha_{1}\right|$ is odd and $\left|\alpha_{3}\right|$ is even.

(By contradiction) Suppose now that $\alpha_{1} \alpha_{2}=0$, since $\alpha_{1}^{2}=\alpha_{2}^{2}=0$ then $\left\{a_{i}, i=1 \cdots 4\right\} \subset V$. Otherwise, the Poincar duality let us to suppose that $\alpha_{1} \alpha_{6}=\alpha_{2} \alpha_{5}=\mu$ and to conclude that $a_{5}, a_{6} \notin \Lambda\left\{a_{i}, i=1 \cdots 4\right\}$ and that $a_{5}, a_{6}$ are also generators of $(\Lambda V, d)$. So

$$
\begin{aligned}
& \sum_{\left|a_{i}\right| \text { odd }}\left|a_{i}\right| \geq\left|\alpha_{1}\right|+\left|\alpha_{2}\right|+\left|\alpha_{5}\right|+\left|\alpha_{6}\right| \\
& =4 f d(X)>2 f d(X)-1 .
\end{aligned}
$$

This is impossible (QED).

Lemma 4 If $\left|\alpha_{1}\right|=\left|\alpha_{2}\right|<\left|\alpha_{3}\right|=\left|\alpha_{4}\right|<\left|\alpha_{5}\right|=\left|\alpha_{6}\right|$, then there exists an homogeneous generator $b$ of $\Lambda V$, satisfying $d b=a_{4}^{2}-\lambda a_{1} a_{2} a_{4}$, where $\lambda \in \mathbb{Q}^{*}$.

Proof. Since $\alpha_{1} \alpha_{2} \neq 0$, we can assume that $a_{1} a_{2}=a_{3}$ and that $a_{4} \in V$, then $\left[a_{3} a_{4}\right]=\alpha_{1} \alpha_{2} \alpha_{4}=\mu$. Otherwise $\left[a_{4}^{2}\right]=\lambda\left[a_{1} a_{2} a_{4}\right]$, then there exists an homogeneous generator $b$ of $\Lambda V$, such $d b=a_{4}^{2}-\lambda a_{1} a_{2} a_{4}$.

Lemma 5 If $\left|\alpha_{1}\right|=\left|\alpha_{2}\right|<\left|\alpha_{3}\right|=\left|\alpha_{4}\right|<\left|\alpha_{5}\right|=\left|\alpha_{6}\right|$, then $(\Lambda V, d) \cong\left(\Lambda\left(x_{1}, x_{2}, x_{3}, y\right), D\right)$ with:

- $D x_{1}=D x_{2}=D y=0$,

- $D y=y^{2}-\lambda x_{1} x_{2} y$ where $\lambda \in \mathbb{Q}^{*}$,

- $\left|x_{1}\right|=\left|x_{2}\right|=\left|a_{1}\right|$ et $|y|=\left|a_{4}\right|$.

Proof. We have $H^{*}(\Lambda V, d) \cong H^{*}(\Lambda W, D)$ where $\Lambda W=\Lambda\left(x_{1}, x_{2}, x_{3}, y\right)$. We define the algebra homomorphism $\Psi:(\Lambda W, D) \rightarrow(\Lambda V, d)$ as $\Psi\left(x_{1}\right)=a_{1}, \Psi\left(x_{2}\right)=a_{2}$, $\Psi\left(x_{3}\right)=b$ and $\Psi(y)=a_{4} . \Psi$ is into because it transforms the basis $\left\{x_{1}, x_{2}, x_{3}, y\right\}$ of $W$ on a the linearly independent family $\left\{a_{1}, a_{2}, a_{4}, b\right\}$. Let $V_{0}=\Psi(W)$ and $V=V_{0} \oplus V_{1}$, since $H^{*}(\Lambda V, d)=H^{*}\left(\Lambda V_{0}, d\right)$ then $d V_{1} \subset \Lambda V_{0} \otimes \Lambda V_{1} \backslash\{0\}$. Assume that $V_{1} \neq 0$ and consider $c \in V_{1}$ such that $|c|=\min \left\{|x|, x \in V_{1}, x \neq 0\right\}$, then $d c=\omega_{1} b+\omega_{2}$ where $\omega_{1}, \omega_{2} \in \Lambda\left(a_{1}, a_{2}, a_{4}\right)$. As $0=d^{2} c=\omega_{1} d b=\omega_{2}\left(a_{4}^{2}-\lambda a_{1} a_{2} a_{4}\right)$, then $\omega_{1}=0$. We have to discuss two cases:

- $d c=\left(\lambda_{1} a_{1}+\lambda_{2} a_{2}\right) a_{4}^{n} ; n \geq 2$. In this case

$|c|=(2 n+1)\left|a_{1}\right|-1$ and therefore

$$
\begin{aligned}
\sum_{\left|a_{i}\right| \text { even }}\left|a_{i}\right| & \geq|b|+|c| \geq 4\left|a_{1}\right|+(2 n+1)\left|a_{1}\right|-2 \\
& \geq 9\left|a_{1}\right|-2>f d(X) .
\end{aligned}
$$

This is impossible.

- $d c=\lambda_{3} a_{1} a_{2} a_{4}^{m}, m \geq 1$. In this case, $|c|=(2 m+3)\left|a_{1}\right|-1$ and $d\left(a_{1} c\right)=0$, then $\left|a_{1} c\right|>5\left|a_{1}\right|-1>f d(X)$, and so $\left[a_{1} c\right]=0$. Let $\beta=\beta_{1}+\cdots+\beta_{k} \in \Lambda V$, such $d \beta=a_{1} c$ where $\beta_{i} \in \Lambda^{i} V$ and $\left|\beta_{1}\right|=(2 m+3)\left|a_{1}\right|-2$ is odd, in particular. But $\beta_{1} \neq 0$, because if not we will have

$$
\begin{aligned}
\sum_{\left|a_{i}\right| \text { odd }}\left|a_{i}\right| & \geq\left|a_{1}\right|+\left|a_{2}\right|+|c|+\left|\beta_{1}\right| \geq(4 m+7)\left|a_{1}\right|-3 \\
& \geq 11\left|a_{1}\right|-3>2 f d(X)-1 .
\end{aligned}
$$

This is impossible.

Proposition 6 If $\left|\alpha_{1}\right|=\left|\alpha_{2}\right|<\left|\alpha_{3}\right|=\left|\alpha_{4}\right|<\left|\alpha_{5}\right|=\left|\alpha_{6}\right|$, then $X$ have one of the following r.h.t:

- $\mathbb{S}^{n} \times \mathbb{S}^{n} \times \mathbb{S}^{2 n}$, where $n$ is odd and $f d(X)=4 n$.

- $\left(\mathbb{S}^{n} \times \mathbb{S}^{n} \times \mathbb{S}^{2 n}\right) \# \mathbb{S}_{(2)}^{2 n}$, where $n$ is odd and $f d(X)=4 n$. Proof. Let us recall that $f d(X)$ and $\left|\alpha_{4}\right|$ are even, and that $\left|\alpha_{1}\right|$ and $\left|\alpha_{6}\right|$ are odd.

- First case: $\alpha_{4}^{2}=0$. Since $\mu=\lambda_{1} \alpha_{1} \alpha_{2} \alpha_{4}$ and $\lambda_{1} \neq 0$, then $\alpha_{1} \alpha_{4} \neq 0$ and $\alpha_{2} \alpha_{4} \neq 0$. Hence

$\left\{1, \alpha_{1}, \alpha_{2}, \alpha_{4}, \alpha_{1} \alpha_{2}, \alpha_{1} \alpha_{4}, \alpha_{2} \alpha_{4}, \alpha_{1} \alpha_{2} \alpha_{4}\right\}$ is a basis for $H^{*}(X ; \mathbb{Q})$, and therefore

$H^{*}(X ; \mathbb{Q}) \cong \mathbb{Q}[a, b, c] /\left(a^{2}, b^{2}, c^{2}\right)$, i.e., $X$ has the r.h.t of $\mathbb{S}^{n} \times \mathbb{S}^{n} \times \mathbb{S}^{2 n}$.

- Second case: $\alpha_{4}^{2} \neq 0$. Here $\alpha_{1} \alpha_{4}$ and $\alpha_{2} \alpha_{4}$ are both non null, because in the opposite case we will have $a_{1} a_{4}=d b$ or $a_{2} a_{4}=d b$ where $b$ is a generator of $\Lambda V$, and in this cases

$\sum_{\left|a_{i}\right| \text { odd }}\left|a_{i}\right| \geq\left|a_{1}\right|+\left|a_{2}\right|+\left|a_{5}\right|+\left|a_{6}\right|>2 f d(X)-1$. This is impossible. Recap $\alpha_{1}^{2}=\alpha_{2}^{2}=0, \alpha_{1} \alpha_{2} \neq 0, \alpha_{4} \neq 0$, $\alpha_{1} \alpha_{4} \neq 0, \alpha_{4} \alpha_{4} \neq 0$, this leads us to conclude that $X$ have the r.h.t of $\left(\mathbb{S}^{n} \times \mathbb{S}^{n} \times \mathbb{S}^{2 n}\right) \# \mathbb{S}_{(2)}^{2 n}$.

\subsection{The Case Where}

$$
\left|\alpha_{1}\right|=\left|\alpha_{2}\right|<\left|\alpha_{3}\right|<\left|\alpha_{4}\right|<\left|\alpha_{5}\right|=\left|\alpha_{6}\right|
$$

Proposition 7 If $\left|\alpha_{1}\right|=\left|\alpha_{2}\right|<\left|\alpha_{3}\right|<\left|\alpha_{4}\right|<\left|\alpha_{5}\right|=\left|\alpha_{6}\right|$ and if $f d(X)$ is even, then $X$ have the r.h.t of $\mathbb{S}^{2 k+1} \times$ $\mathbb{S}^{2 k+1} \times \mathbb{S}^{2(k+p)}$ with $f d(X)=2(k+1)+2(k+p)$.

Proof. Because of the parity of $f d(X)$, the duality of Poincar and the fact that $\chi_{c}=0$, then $\left|\alpha_{1}\right|$ and $\left|\alpha_{3}\right|$ are respectively odd and even, so $\alpha_{1}^{2}=\alpha_{2}^{2}=0$. Assume that $\alpha_{2} \alpha_{2}=0$ and that $\alpha_{5}, \alpha_{6} \in \Lambda\left(\alpha_{1}, \alpha_{2}, \alpha_{3}, \alpha_{4}\right)$, then there exist $P_{1}^{i}, P_{2}^{i} \in \Lambda\left(\alpha_{1}, \alpha_{2}, \alpha_{3}, \alpha_{4}\right)$ such that $\alpha_{i}=P_{1}^{i} \alpha_{1}+P_{2}^{i} \alpha_{2}$ for $i=5,6$. This implies the impossible situation that $\mu=\alpha_{1} \alpha_{i}=\alpha_{2} \alpha_{i}=0$, but also that our second assumption is false. Thus necessarily $a_{5}, a_{6}$ are both generators of $\Lambda V$ and that

$\sum_{\left|a_{i}\right| \text { odd }}\left|a_{i}\right| \geq\left|a_{1}\right|+\left|a_{2}\right|+\left|a_{5}\right|+\left|a_{6}\right| \geq 2 f d(X)>2 f d(X)-1$.

This another impossible situation implies that our first assumption is also false. Put $\alpha_{1} \alpha_{3} \neq 0$, in this case $\left\{a_{3}, a_{4}\right\} \cap \mathbb{Q}\left\{a_{1}, a_{2}\right\} \neq \varnothing$, in particular $a_{j}$ are generators of $\Lambda V$ for $j=3,4$. The Poincar duality let us to write $\alpha_{1} \alpha_{2} \alpha_{j}=\left[a_{1} a_{2} a_{j}\right]=\mu$ and to conclude that $\alpha_{1} \alpha_{j} \neq 0$ and that $\alpha_{2} \alpha_{j} \neq 0$ and finally to write $\alpha_{5}=\alpha_{1} \alpha_{j}, \alpha_{6}=\alpha_{2} \alpha_{j}$. Recall that $\alpha_{j}^{2}=0$, because of the parity of the degree, then $(\Lambda V, d) \cong \Lambda(a, b, c, x), d)$ with $d a=d b=d c=0$ and $d x=c^{2}$. This is the minimal model of $\mathbb{S}^{2 k+1} \times \mathbb{S}^{2 k+1} \times \mathbb{S}^{2(k+p)}$. 
Proposition 8 If $\left|\alpha_{1}\right|=\left|\alpha_{2}\right|<\left|\alpha_{3}\right|<\left|\alpha_{4}\right|<\left|\alpha_{5}\right|=\left|\alpha_{6}\right|$ and if $f d(X)$ is odd, then $X$ has one of following r.h.t:

- $\mathbb{S}^{2 k+1} \times \mathbb{S}^{2 k+1} \times \mathbb{S}^{2(k+p)}$ with $f d(X)=2(k+1)+2(k+p)$,

- $\left(\mathbb{S}^{2 n} \otimes_{d_{2}} \mathbb{S}^{2 n}\right) \times \mathbb{S}^{2(n+p)+1}$ with $f d(X)=4 n+2(n+p)+1$,

- $\left(\mathbb{S}^{2 n} \times \mathbb{S}^{2 n}\right) \times \mathbb{S}^{2(n+p)+1}$ with $f d(X)=4 n+2(n+p)+1$,

Proof. We will discuss three cases:

- First case: $\left|\alpha_{1}\right|$ is odd, then $\left|\alpha_{5}\right|$ is even. Suppose that $\left|\alpha_{5}\right| \in V^{\text {even }}$, then

$\sum_{a_{i} \mid \text { even }}\left|a_{i}\right| \geq\left|a_{3}\right|+\left|a_{5}\right|>\left|a_{1}\right|+\left|a_{5}\right|=f d(X)$. This is impossible. So $\left\{\alpha_{3}, \alpha_{4}\right\} \cap \mathbb{Q}\left\{\alpha_{1}, \alpha_{2}\right\}$ with $\alpha_{1} \alpha_{2} \neq 0$ and $a_{j}$ is an odd degree generator of $\Lambda V$ for $j=3$ or $j=4$. A same justification as in the last proof let us to conclude that $\alpha_{5}=\alpha_{1} \alpha_{j}, \alpha_{6}=\alpha_{2} \alpha_{j}$ and that $X$ have the r.h.t of $\mathbb{S}^{2 k+1} \times \mathbb{S}^{2 k+1} \times \mathbb{S}^{2(k+p)}$.

- Second case: $\left|\alpha_{1}\right|$ is even and $\alpha_{1} \alpha_{2}=0$. Since $\left|\alpha_{5}\right|$ is odd, then $\operatorname{dim} \mathbb{Q}\left\{\alpha_{1}^{2}, \alpha_{2}^{2}, \alpha_{1} \alpha_{2}\right\} \leq 1$. Assume for example that $\alpha_{1}^{2} \neq 0$, then $\mu=\alpha_{1}^{2} \alpha_{j}$ with $j=3$ or 4 and $\left|\alpha_{j}\right|$ is odd. Therefore $\alpha_{1} \alpha_{j} \neq 0$. Let suppose that $\alpha_{1} \alpha_{j}, \alpha_{2} \alpha_{j}$ are collinear and write $\alpha_{2} \alpha_{j}=$ $\lambda \alpha_{1} \alpha_{j}$, then $\alpha_{2}^{2} \alpha_{j}=\lambda \alpha_{2} \alpha_{1} \alpha_{j}=0$, so $a_{5} \in V^{\text {odd }}$. Since that $\alpha_{1} \alpha_{2}=0$ and that $\alpha_{1}^{2}=\beta \alpha_{2}^{2}$, then there exist two odd degree generators of $\Lambda V, b$ and $c$, such that $d b=a_{1} a_{2}$ and $d c=a_{1}^{2}-\beta a_{2}^{2}$. We conclude that

$$
\begin{aligned}
\sum_{\left|a_{i}\right| \text { odd }}\left|a_{i}\right| & \geq\left|a_{j}\right|+\left|a_{5}\right|+|b|+|c| \geq\left|a_{j}\right|+\left|a_{5}\right|+2\left(2\left|a_{1}\right|-1\right) \\
& \geq 2 f d(X)-1+\left|a_{1}\right|-1>2 f d(X)-1
\end{aligned}
$$

(impossible). Put $\alpha_{1} \alpha_{j}=\alpha_{6}$ and $\alpha_{2} \alpha_{j}=\alpha_{5}$, then $\mu=\alpha_{1}^{2} \alpha_{j}=\alpha_{2}^{2} \alpha_{j}$ and $\alpha_{1}^{2} \neq 0, \alpha_{2}^{2} \neq 0$. The minimal model of $X$ will be of the form

$(\Lambda V, d) \cong\left(\Lambda\left(a_{1}, a_{2}, b_{1}, b_{2}, b_{3}\right), d\right)$ with $d a_{1}=d a_{2}=0$, $d b_{1}=a_{1} a_{2}, d b_{2}=a_{2}^{2}-\lambda a_{1}^{2}, d b_{3}=0$ and $\left|a_{1}\right|=2 n$, $|b|=2(n+p)+1$, i.e., $\quad X \sim\left(\mathbb{S}^{2 n} \otimes_{d_{2}} \mathbb{S}^{2 n}\right) \times \mathbb{S}^{2(n+p)+1}$.

- Third case: $\left|\alpha_{1}\right|$ is even and $\alpha_{1} \alpha_{2} \neq 0$. As in the first case, we can write $\alpha_{5}=\alpha_{1} \alpha_{j}, \alpha_{6}=\alpha_{2} \alpha_{j}$. Since $\operatorname{dim} \mathbb{Q}\left\{\alpha_{1}^{2}, \alpha_{2}^{2}, \alpha_{1} \alpha_{2}\right\} \leq 1$, then $\alpha_{1}^{2}=\lambda_{1} \alpha_{1} \alpha_{2}$ and $\alpha_{2}^{2}=$ $\lambda_{2} \alpha_{1} \alpha_{2}$. Suppose that $\lambda_{1} \neq 0$ and write $a_{1}^{3}=d a$, $a_{1}^{2}-\lambda_{1} a_{1} a_{2} \quad\left(\alpha_{1}^{3}=0\right)$, then $d\left(\frac{1}{\lambda_{1}}\left(a-a_{1} b\right)\right)$, i.e., $\alpha_{1} \alpha_{2}=0$. That contradicts the main hypothesis in our third case. Hence the minimal model of $X$ will be of the form $(\Lambda V, d) \cong\left(\Lambda\left(a_{1}, a_{2}, b, b_{1}, b_{2}\right), d\right)$ with $d a_{1}=d a_{2}=d b=0, d b_{1}=a_{1}^{2}, d b_{2}=a_{2}^{2}$, i.e., $X \sim \mathbb{S}^{2 n} \times \mathbb{S}^{2 n} \times \mathbb{S}^{2(n+p)+1}$.

\subsection{The Case Where}

$$
\left|\alpha_{1}\right|<\left|\alpha_{2}\right|=\left|\alpha_{3}\right|<\left|\alpha_{4}\right|=\left|\alpha_{5}\right|<\left|\alpha_{6}\right|
$$

Proposition 9 If $\left|\alpha_{1}\right|<\left|\alpha_{2}\right|=\left|\alpha_{3}\right|<\left|\alpha_{4}\right|=\left|\alpha_{5}\right|<\left|\alpha_{6}\right|$ and $f d(X)$ is even, then $x$ have one the r.h.t of $\mathbb{S}^{2 n} \times\left(\mathbb{S}^{2(n+p)+1}\right)^{2}$.

Proof. As $f d(X)$ is even and $\chi_{c}=0$, then $\left|\alpha_{1}\right|$ and $\left|\alpha_{2}\right|$ are respectively even and odd. Suppose (by contradiction) that $\alpha_{1} \alpha_{2}$ or $\alpha_{1} \alpha_{3}$ is null (for example $\alpha_{1} \alpha_{2}=0$ ). The duality of Poincar insures that $\left\{a_{4}, a_{5}\right\} \subset V$, then $\sum_{\mid a_{i} \text { odd }}\left|a_{i}\right| \geq\left|a_{2}\right|+\left|a_{3}\right|+\left|a_{4}\right|+\left|a_{5}\right|>2 f d(X)-1$. This is impossible (QED). Put $\alpha_{4}=\alpha_{1} \alpha_{2}, \alpha_{5}=\alpha_{1} \alpha_{3}$, then $\alpha_{2} \alpha_{3} \neq 0$, because that $\mu=\alpha_{1} \alpha_{2} \alpha_{3}$. This leads us to take $\alpha_{6}=\alpha_{2} \alpha_{3}$ and to conclude that $\alpha_{1}^{2}=0$. Hence $(\Lambda V, d) \cong\left(\Lambda\left(x, y_{1}, y_{2}, y\right), d\right)$ with $d x=d y_{1}=d y_{2}=0$, $d y=x^{2}$ and $|x|=2 n,\left|y_{1}\right|=\left|y_{2}\right|=2(n+p)+1$, i.e., $X \sim \mathbb{S}^{2 n} \times\left(\mathbb{S}^{2(n+p)+1}\right)^{2}$.

Lemma 10 If $\left|\alpha_{1}\right|<\left|\alpha_{2}\right|=\left|\alpha_{3}\right|<\left|\alpha_{4}\right|=\left|\alpha_{5}\right|<\left|\alpha_{6}\right|$ and $f d(X)$ is odd, then $\left\{a_{4}, a_{5}\right\} \cap V=\varnothing$.

Let us suppose $a_{4} \in V$ (for example) and discuss two cases:

- $\left|a_{4}\right|$ is even, then $\alpha_{4}^{2}=0$ and there exists a generator $a_{7}$ of $\Lambda V$ such that $d a_{7}=a_{4}^{2}$. If $\left|a_{1}\right|$ is odd, then $\sum_{\mid a_{i} \text { odd }}\left|a_{i}\right| \geq\left|a_{1}\right|+\left|a_{2}\right|+\left|a_{3}\right|+\left|a_{7}\right|>2 f d(X)-1$, impossible. Then $\left|a_{1}\right|$ is even and necessary $\operatorname{dim} \mathbb{Q}\left\{\alpha_{1}^{2}, \alpha_{2} \alpha_{3}\right\} \leq 1$, i.e., there exists a generator $a_{8}$ of $\Lambda V$ verifying $d a_{8}=\lambda_{1} a_{1}^{2}+\lambda_{2} a_{2} a_{3}$ with $\lambda_{1}=0$ or $\lambda_{2}=0$. Consequently

$$
\begin{aligned}
\sum_{\left|a_{i}\right| \text { odd }}\left|a_{i}\right| & \geq\left|a_{2}\right|+\left|a_{3}\right|+\left|a_{7}\right|+\left|a_{8}\right| \\
& \geq\left|a_{2}\right|+\left|a_{3}\right|+\left|a_{7}\right|+\left|a_{8}\right| \\
& \geq 2\left|a_{2}\right|+2\left|a_{4}\right|-1+2\left|a_{1}\right|-1 \\
& >2\left(\left|a_{2}\right|+\left|a_{4}\right|\right)-1=2 f d(X)-1,
\end{aligned}
$$

what is, once again, an impossible situation.

- $\left|a_{4}\right|$ is odd, because of the Poincar duality, we must have $\left|a_{2}\right|$ be even and $\operatorname{dim} \mathbb{Q}\left\{\alpha_{2} \alpha_{3}, \alpha_{3} \alpha_{4}\right\}=1$. Let $a_{9} \in \Lambda V$ such that $d a_{9}=\lambda_{1} a_{2} a_{4}-\lambda_{2} a_{3} a_{4}$, then

$$
\begin{aligned}
\sum_{\left|a_{i}\right| \text { even }}\left|a_{i}\right| & \geq\left|a_{2}\right|+\left|a_{3}\right|+\left|a_{9}\right| \geq 2\left|a_{2}\right|+\left|a_{2}\right|+\left|a_{4}\right|-1 \\
& >\left|a_{2}\right|+\left|a_{4}\right|=f d(X) .
\end{aligned}
$$

This is impossible.

Lemma 11 If $\left|\alpha_{1}\right|<\left|\alpha_{2}\right|=\left|\alpha_{3}\right|<\left|\alpha_{4}\right|=\left|\alpha_{5}\right|<\left|\alpha_{6}\right|$ and $f d(X)$ is odd, then $\alpha_{1} \alpha_{2} \neq 0$ and $\alpha_{1} \alpha_{3} \neq 0$.

Proof. (By contradiction) Assume, for example, that $\alpha_{1} \alpha_{2}=0$. By the precedent lemma and the duality of Poincar, we have $\left\{a_{4}, a_{5}\right\} \subset \Lambda\left\{a_{1}, a_{2}, a_{3}\right\}$ and $\alpha_{2} \alpha_{5} \neq 0$, $\alpha_{3} \alpha_{4} \neq 0$. Therefore $\alpha_{5} \in \mathbb{Q}\left\{\alpha_{2}^{2}, \alpha_{3}^{2}, \alpha_{2} \alpha_{3}\right\}$, but $f d(X)$ is odd, then $\alpha_{5} \in \mathbb{Q} \alpha_{2} \alpha_{3}$ and $\mu \in \mathbb{Q} \alpha_{2} \alpha_{5}=\mathbb{Q} \alpha_{2}^{2} \alpha_{3}=0$. This is a contradiction $(Q E D)$.

Proposition 12 If $\left|\alpha_{1}\right|<\left|\alpha_{2}\right|=\left|\alpha_{3}\right|<\left|\alpha_{4}\right|=\left|\alpha_{5}\right|<\left|\alpha_{6}\right|$ and $f d(X)$ is odd, then $x$ have one of the following r.h.t:

- $\mathbb{S}^{2 n+1} \times \mathbb{S}^{k} \times \mathbb{S}^{k}$ with $k \geq 2 n+2$,

- $\mathbb{S}^{2 n+1} \times Y_{\lambda}$, where $\lambda \in \mathbb{Q}^{*}$ and $Y_{\lambda}$ have a minimal 
model of the form $(\Lambda(a, b, u, v), d)$ with $d a=d b=0$, $d u=a b, d v=b^{2}-\lambda a^{2}$.

Proof. By the two last lemmas, we have $\left|\alpha_{1}\right|$ is odd and $(\Lambda V, d) \cong\left(\Lambda a_{1}, 0\right) \otimes\left(\Lambda V^{\prime}, d\right)$ with $\left\{a_{2}, a_{3}\right\} \in V^{\prime}$. But $\operatorname{dim} H^{*}\left(\Lambda V^{\prime}, d\right)=4$ (case classified by the first author in his thesis), then $X \sim \mathbb{S}^{n_{1}} \times Y$ where $Y \sim \mathbb{S}^{n_{1}} \times \mathbb{S}^{n_{2}}$ and $n_{2} \geq n_{1}+1$ or $Y \sim Y_{\lambda}$ where $\left(\Lambda V^{\prime}, d\right) \cong(\Lambda(a, b, u, v), d)$ and $d a=d b=0, d u=a b$, $d v=b^{2}-\lambda a^{2}$.

\subsection{Case Where}

$$
\left|\alpha_{1}\right|<\left|\alpha_{2}\right|<\left|\alpha_{3}\right|=\left|\alpha_{4}\right|<\left|\alpha_{5}\right|<\left|\alpha_{6}\right|
$$

Lemma 13 If $\left|\alpha_{1}\right|<\left|\alpha_{2}\right|<\left|\alpha_{3}\right|=\left|\alpha_{4}\right|<\left|\alpha_{5}\right|<\left|\alpha_{6}\right|$, then $a_{3} \in \Lambda\left(a_{1}, a_{2}\right)$ or $a_{4} \in \Lambda\left(a_{1}, a_{2}\right)$.

Proof. Suppose that $\left\{a_{3}, a_{4}\right\} \in V$, then there exist two generators $a_{7}$ and $a_{8}$ of $\Lambda V$ satisfying

$d a_{7}=a_{3} a_{4}-\lambda a_{2} a_{5}+\omega$ and $d a_{8}=\lambda_{1} a_{1} a_{3}+\lambda_{2} a_{1} a_{4}$ with $\omega \in \Lambda^{\geq 3} V$. We distinguish two cases:

- First case: $\left|\alpha_{3}\right|$ is even, then $\mid \alpha_{1}$ is odd. As $f d(X)$ is even and $\chi_{c}=0$, then $\mid \alpha_{8}$ is even and consequently $\sum_{\left|a_{i}\right| \text { even }}\left|a_{i}\right| \geq\left|a_{3}\right|+\left|a_{4}\right|+\left|a_{8}\right|>f d(X)$.

- Second case: $\left|\alpha_{3}\right|$ is odd. As $f d(X)$ is even and $\chi_{c}=0$, then $\left|a_{8}\right|$ is even and

$\sum_{\left|a_{i}\right| \text { even }}\left|a_{i}\right| \geq\left|a_{3}\right|+\left|a_{4}\right|+\left|a_{8}\right|>f d(X)$.

The two cases are both impossible.

Lemma 14 If $\left|\alpha_{1}\right|<\left|\alpha_{2}\right|<\left|\alpha_{3}\right|=\left|\alpha_{4}\right|<\left|\alpha_{5}\right|<\left|\alpha_{6}\right|$, then:

1) $\alpha_{1}^{2}=0$

2) $\left\{a_{3}, a_{4}\right\} \cap \mathbb{Q}\left\{a_{1} a_{2}\right\} \neq \varnothing$,

3) $\left\{a_{3}, a_{4}\right\} \cap V \neq \varnothing$,

4) $\alpha_{5} \in \mathbb{Q}^{*} \alpha_{1} \alpha_{4}$ and $\alpha_{6} \in \mathbb{Q}^{*} \alpha_{2} \alpha_{4}$.

Proof. 1) suppose that $\alpha_{1}^{2}=0$, then $\left|\alpha_{1}\right|$ is even. Since $f d(X)$ is even and $\chi_{c}=0$, then $\left|\alpha_{2}\right|,\left|\alpha_{3}\right|$ and $\left|\alpha_{5}\right|$ are both odd. Put $\alpha_{6}=\alpha_{1}^{2}$, then $\left\{a_{2}, a_{3}, a_{4}, a_{4}\right\} \subset V$ and $\sum_{\left|a_{i}\right| \text { odd }}\left|a_{i}\right| \geq\left|a_{2}\right|+\left|a_{3}\right|+\left|a_{4}\right|+\left|a_{5}\right|>2 f d(X)-1$ (contradiction).

2) We have $\left\{a_{3}, a_{4}\right\} \cap \mathbb{Q}\left\{a_{1} a_{2}, a_{2}^{2}\right\} \neq \varnothing$. If $\left|\alpha_{2}\right|$ is even, then $\left|\alpha_{3}\right|$ is odd and $\left\{a_{3}, a_{4}\right\} \cap \mathbb{Q}\left\{a_{1} a_{2}\right\} \neq \varnothing$. If $\left|\alpha_{2}\right|$ is odd, the result is evident because that $\alpha_{2}^{2}=0$.

3 ) It is an immediate consequence of 2). Hence we can take $\alpha_{6}=\alpha_{1}^{2}$ and $\left\{a_{2}, a_{3}, a_{4}, a_{5}\right\} \subset V^{\text {odd }}$.

4) Since $\alpha_{3}^{2}=\alpha_{1}^{2} \alpha_{2}^{2}=0$, then there exists $\lambda \in \mathbb{Q}^{*}$ such that $\alpha_{3} \alpha_{4}=\lambda \mu$. So $\alpha_{1} \alpha_{4} \neq 0, \alpha_{2} \alpha_{4} \neq 0$ and $\alpha_{5} \in \mathbb{Q}^{*} \alpha_{1} \alpha_{4}, \alpha_{6} \in \mathbb{Q}^{*} \alpha_{2} \alpha_{4}$.

Proposition 15 If $\left|\alpha_{1}\right|<\left|\alpha_{2}\right|<\left|\alpha_{3}\right|=\left|\alpha_{4}\right|<\left|\alpha_{5}\right|<\left|\alpha_{6}\right|$, then $X$ have the h.r.t of $\mathbb{S}^{2 n} \times \mathbb{S}^{2 k+1} \times \mathbb{S}^{2(n+k)+1}$ or that of $\mathbb{S}^{2 n+1} \times \mathbb{S}^{2(n+k)+1} \otimes_{d_{2}} \mathbb{S}^{2 k}$.

Proof. Put $\alpha_{5}=\alpha_{1} \alpha_{4}, \alpha_{6}=\alpha_{2} \alpha_{4}$ and $\alpha_{2}^{2}=\lambda_{1} \alpha_{2} \alpha_{4}$, $\alpha_{4}^{2}=\lambda_{2} \alpha_{1} \alpha_{2} \alpha_{4}$, then the minimal model of $X$ have one of the following forms:

- $(\Lambda V, d) \cong\left(\Lambda\left(x, y_{1}, y_{2}, y_{3}\right), d\right)$ with

$$
d x_{1}=d y_{1}=d y_{2}=0, d y=x_{1}^{2} \text { and }\left|x_{1}\right|=2 n \text {, }
$$

$\left|y_{1}\right|=2 k+1,\left|y_{2}\right|=2(k+n)+1$, i.e., $X \sim \mathbb{S}^{2 n} \times \mathbb{S}^{2 k+1} \times \mathbb{S}^{2(n+k)+1}$.

- $(\Lambda V, d) \cong\left(\Lambda\left(x, y_{1}, y_{2}, y_{3}\right), d\right)$ with $d x_{1}=d y_{1}=d y_{2}=0, \quad d y_{3}=x^{2}-\lambda y_{1} y_{2}$ and $\left|x_{1}\right|=2 k$, $\left|y_{1}\right|=2 n+1,\left|y_{2}\right|=2(k+n)+1$, i.e., $X \sim \mathbb{S}^{2 n+1} \times \mathbb{S}^{2(n+k)+1} \otimes_{d_{2}} \mathbb{S}^{2 k}$.

\subsection{Case Where}

$$
\left|\alpha_{1}\right|<\left|\alpha_{2}\right|<\left|\alpha_{3}\right|<\left|\alpha_{4}\right|<\left|\alpha_{5}\right|<\left|\alpha_{6}\right|
$$

Lemma 16 If $\left|\alpha_{1}\right|<\left|\alpha_{2}\right|<\left|\alpha_{3}\right|<\left|\alpha_{4}\right|<\left|\alpha_{5}\right|<\left|\alpha_{6}\right|$, then $a_{5} \in \Lambda\left(a_{1}, a_{2}, a_{3}, a_{4}\right)$.

Proof. Let $a_{5} \in V$ and discuss many cases:

1) $a_{5} \in V^{\text {even }}$, then there exist two generators $x$ and $y$ of $\Lambda V$ such that $d x=a_{2} a_{5}-a_{3} a_{4}$ and $d y=a_{5}^{2}$

a) $\left|a_{2}\right|$ is odd, then

$$
\sum_{\left|a_{i}\right| \text { even }}\left|a_{i}\right| \geq\left|a_{1}\right|+\left|a_{2}\right|+\left|a_{6}\right|+\left|a_{9}\right|>f d(X) .
$$

b) $\left|a_{2}\right|$ is even. As $f d(X)$ is even and $\chi_{c}=0$, then $\mid a_{1}$ is odd, $a_{1} \in V^{\text {even }}$ and

$$
\sum_{\left|a_{i}\right| \text { odd }}\left|a_{i}\right| \geq|x|+|y|>2 f d(X)-1 .
$$

2) $a_{5} \in V^{\text {odd }}$.

a) $\left|a_{2}\right|$ is odd, then necessary $a_{2} \in V^{\text {odd }}$ and $\alpha_{2} \alpha_{5}=$ $\alpha_{3} \alpha_{4}=\mu$. Hence there exists a generator $x$ of $\Lambda V$ such that $d x=a_{2} a_{5}-a_{3} a_{4}$.

i) $a_{1} \in V^{\text {even }}$, then $\left|a_{3}\right|$ and $\mid a_{2}$ are both odd since $\chi_{c}=0$. Since $\alpha_{3} \alpha_{4} \neq 0$ then $a_{j} \in V^{\text {odd }}$ for $j=3$ or $j=4$ with $\alpha_{i} \alpha_{5}=0$. Hence there exists a generator $y$ of $\Lambda V$ such that $d y=a_{j} a_{5}$ and so

$$
\sum_{\left|a_{i}\right| \text { odd }}\left|a_{i}\right| \geq|x|+|y|>2 f d(X)-1 .
$$

ii) $a_{1} \in V^{\text {odd }}$, then $x \in V^{\text {odd }}$ and

$$
\sum_{\left|a_{i}\right| \text { odd }}\left|a_{i}\right| \geq\left|a_{1}\right|+\left|a_{2}\right|+\left|a_{3}\right|+|x|>2 f d(X)-1 .
$$

b) $\left|a_{2}\right|$ is even.

i) $a_{2} \in V^{\text {even }}$, then necessary $x \in V^{\text {even }}$ and

$$
\sum_{\left|a_{i}\right| \text { even }}\left|a_{i}\right| \geq\left|a_{2}\right|+|x|>f d(X) .
$$

ii) $a_{2}=a_{1}^{2}$, then $\alpha_{1}^{2} \alpha_{5} \neq 0$ (because of the Poincar duality) and $\alpha_{1} \alpha_{5} \neq 0$. Put $a_{6}=a_{1} a_{5}$, then $a_{j} \in V^{\text {odd }}$ for $j=3$ or $j=4 \quad\left(\chi_{c}=0\right)$ and $\alpha_{j} \alpha_{5}=0$ (Poincar duality). Let $x$ be a generator of $\Lambda V$ such that $d x=a_{j} a_{5}$, then

$$
\begin{aligned}
\sum_{\left|a_{i}\right| \text { odd }}\left|a_{i}\right| & \geq\left|a_{j}\right|+\left|a_{5}\right|+|x| \\
& \geq 2\left(\left|a_{j}\right|+\left|a_{5}\right|\right)-1>2 f d(X)-1 .
\end{aligned}
$$

Lemma 17 If $\left|\alpha_{1}\right|<\left|\alpha_{2}\right|<\left|\alpha_{3}\right|<\left|\alpha_{4}\right|<\left|\alpha_{5}\right|<\left|\alpha_{6}\right|$, then $a_{6} \in \Lambda\left(a_{1}, a_{2}, a_{3}, a_{4}\right)$.

Proof. Let $a_{6} \in V$ and discuss many cases:

1) $a_{6} \in V^{\text {even }}$. Then there exist generators $a_{7}, a_{8}$ of 
$\Lambda V$ such that $d a_{7}=a_{1} a_{6}-a_{3} a_{4}$ and $d a_{8}=a_{6}^{2}$.

a) $\left|a_{1}\right|$ is even, then

$$
\begin{aligned}
\sum_{\left|a_{i}\right| \text { odd }}\left|a_{i}\right| & \geq\left|a_{7}\right|+\left|a_{8}\right| \geq f d(X)-1+2\left|a_{6}\right|-1 \\
& \geq 2 f d(X)-1+\left|a_{6}\right|-1>2 f d(X)-1 .
\end{aligned}
$$

b) $\left|a_{1}\right|$ is odd, then

$$
\sum_{\left|a_{i}\right| \text { even }}\left|a_{i}\right| \geq\left|a_{6}\right|+\left|a_{7}\right|>f d(X) .
$$

2) $a_{6} \in V^{\text {odd }}$

a) $a_{2} \in V^{\text {even }}$, then

$$
\sum_{\left|a_{i}\right| \text { even }}\left|a_{i}\right| \geq\left|a_{2}\right|+\left|a_{9}\right|>f d(X) .
$$

b) $a_{2} \in V^{\text {odd }}$, then

$$
\sum_{\left|a_{i}\right| \text { odd }}\left|a_{i}\right| \geq\left|a_{1}\right|+\left|a_{2}\right|+\left|a_{6}\right|+\left|a_{9}\right|>2 f d(X)-1 .
$$

Lemma 18 If $\left|\alpha_{1}\right|<\left|\alpha_{2}\right|<\left|\alpha_{3}\right|<\left|\alpha_{4}\right|<\left|\alpha_{5}\right|<\left|\alpha_{6}\right|$, then $2 \leq\left\{a_{1}, a_{2}, a_{3}, a_{4}\right\} \cap V \mid \leq 3$.

Proof. Put $N=\left|\left\{a_{1}, a_{2}, a_{3}, a_{4}\right\} \cap V\right|$.

1) If $N=1$, then $a_{i} \in \Lambda\left(a_{i}\right)$ for all $i=1, \cdots, 6$, this implies the contradiction $\chi_{c} \neq 0$.

2) If $N=4$. We have $\alpha_{3} \alpha_{4}=\alpha_{1} \alpha_{6}=\mu$.

a) $\left|a_{3}\right|$ and $\left|a_{4}\right|$ are both even, then $f d(X)$ is even and $\alpha_{4}^{2}=0$. Let $a_{7}$ and $a_{8}$ be some generators of $\Lambda V$ with $d a_{7}=a_{1} a_{6}-a_{3} a_{4}$ and $d a_{8}=a_{4}^{2}$, therefore

$$
\sum_{\left|a_{i}\right| \text { odd }}\left|a_{i}\right| \geq\left|a_{7}\right|+\left|a_{8}\right|>2 f d(X)-1 .
$$

b) $\left|a_{3}\right|$ and $\left|a_{4}\right|$ are both odd, then $f d(X)$ is odd and $\chi_{c}=2\left((-1)^{\left|a_{1}\right|}+(-1)^{\left|a_{2}\right|}\right)=0$, so $\left|\alpha_{1}\right|$ (for example) is odd and

$$
\sum_{\left|a_{i}\right| \text { odd }}\left|a_{i}\right| \geq\left|a_{1}\right|+\left|a_{3}\right|+\left|a_{4}\right|+\left|a_{7}\right|>2 f d(X)-1 .
$$

c) $\left|a_{3}\right|$ is even and $\left|a_{4}\right|$ is odd (for example), then

$$
\sum_{\left|a_{i}\right| \text { even }}\left|a_{i}\right| \geq\left|a_{3}\right|+\left|a_{7}\right|>f d(X) .
$$

Lemma 19 If $\left|\alpha_{1}\right|<\left|\alpha_{2}\right|<\left|\alpha_{3}\right|<\left|\alpha_{4}\right|<\left|\alpha_{5}\right|<\left|\alpha_{6}\right|$ and if $\left\{a_{1}, a_{2}, a_{3}, a_{4}\right\} \cap V \mid=2$, then $a_{1}$ and $a_{j}$ have different parities where $a_{j}=\left\{a_{2}, a_{3}, a_{4}\right\} \cap V$.

Proof. Suppose that $a_{1}$ and $a_{j}$ have the same parity.

1) If $\left|a_{1}\right|$ and $\left|a_{j}\right|$ are both even, then all $\left|a_{i}\right|$ are even for $i=1, \cdots, 6$ and $\chi_{c} \neq 0$.

2) If $\left|a_{1}\right|$ and $\left|a_{j}\right|$ are odd, then necessary $a_{j}=a_{2}$ and $\left\{\alpha_{3}, \alpha_{4}\right\} \subset \mathbb{Q} \alpha_{1} \alpha_{2}$ (because that $\left.\left\{a_{3}, a_{4}\right\} \subset \Lambda\left(a_{1}, a_{2}\right)\right)$, but this is impossible.

Proposition 20 If $\left|\alpha_{1}\right|<\left|\alpha_{2}\right|<\left|\alpha_{3}\right|<\left|\alpha_{4}\right|<\left|\alpha_{5}\right|<\left|\alpha_{6}\right|$ and if $\left|\left\{a_{1}, a_{2}, a_{3}, a_{4}\right\} \cap V\right|=2$, then $X \sim \mathbb{S}_{(3)}^{n} \times \mathbb{S}^{2 m+1}$.

Proof. Put $\left\{a_{1}, a_{2}, a_{3}, a_{4}\right\} \cap V=\left\{a_{1}, a_{j}\right\}$. Because the duality of Poincar, the fact that $\left|a_{1}\right|,\left|a_{j}\right|$ have different parities and the fact that $a_{i} \in \Lambda\left(a_{1}, a_{j}\right)$ for all $i=1, \cdots, 6$. Let $a_{\ell} \in\left\{a_{1}, a_{j}\right\}$ such that $\left|a_{\ell}\right|$ is odd and $a_{k} \in\left\{a_{1}, a_{j}\right\}-a_{\ell}$, it is evident that $\left|a_{k}\right|$ is even. As $H^{*}(\Lambda V, d)=\mathbb{Q}\left\{\alpha_{\ell}^{m} \alpha_{k}^{n}, n \in \mathbb{N}, m=0,1\right\}$, then $\alpha_{k} \alpha_{7-k}=$ $\alpha_{\ell} \alpha_{7-\ell}=\mu$. This allows us to take

$\left\{a_{7-\ell}, a_{7-k}\right\}=\left\{a_{k}^{p}, a_{\ell} a_{k}^{p-1}\right\}$ with $p=3$, because if not $\operatorname{dim} H^{*}(\Lambda V, d) \neq 8$. Hence $\alpha_{k}^{4}=0$ and $\alpha_{\ell} \alpha_{k}^{n} \neq 0$. Conclude that $(\Lambda V, d) \cong \Lambda\left(a_{\ell}, 0\right) \otimes(\Lambda W, d)$, that $\operatorname{dim} V^{\text {even }}=1$ and that $\operatorname{dim} H^{*}(\Lambda W, d)=4$. In [?], $(\Lambda W, d)$ is the minimal model of $\mathbb{S}_{(3)}^{n}$, then $X \sim \mathbb{S}_{(3)}^{n} \times \mathbb{S}^{2 m+1}$ where $n=\left|a_{k}\right|$ and $2 m+1=\left|a_{\ell}\right|$.

Lemma 21 If $\left|\alpha_{1}\right|<\left|\alpha_{2}\right|<\left|\alpha_{3}\right|<\left|\alpha_{4}\right|<\left|\alpha_{5}\right|<\left|\alpha_{6}\right|$ and if $\left|\left\{a_{1}, a_{2}, a_{3}, a_{4}\right\} \cap V\right|=3$, then only one among $a_{3}$ or $a_{4}$ is in $V$.

Proof. Assume that $\left\{a_{3}, a_{4}\right\} \in V$, then $\left|a_{1}\right|$ and $\left|a_{2}\right|$ are both even, because that necessary $a_{2} \in \mathbb{Q} a_{1}^{2}$. Therefore $\chi_{c}=\left(1+(-1)^{f d(X)}\right)\left(3+(-1)^{\left|a_{3}\right|}\right)=0$, i.e., $f d(X)$ is odd, and there exists a generator $a_{7}$ of $\Lambda V$, such that $d a_{7}=a_{3} a_{4}-a_{1} a_{6}$ with

$$
\sum_{\left|a_{i}\right| \text { even }}\left|a_{i}\right| \geq\left|a_{3}\right|+\left|a_{7}\right|>f d(X) .
$$

Lemma 22 If $\left|\alpha_{1}\right|<\left|\alpha_{2}\right|<\left|\alpha_{3}\right|<\left|\alpha_{4}\right|<\left|\alpha_{5}\right|<\left|\alpha_{6}\right|$ and if $\left|\left\{a_{1}, a_{2}, a_{3}, a_{4}\right\} \cap V\right|=3$, then $a_{j}=a_{1} a_{2}$ where $a_{j}=\left\{a_{3}, a_{4}\right\} \cap V$.

Proof. Suppose $\alpha_{1} \alpha_{2}=0$, we know, from the duality of Poincar, that $\alpha_{1} \alpha_{6}=\alpha_{2} \alpha_{5}=\mu$ and by the Lemmas 16 and 17 that $\left\{a_{5}, a_{6}\right\} \subset \Lambda\left(a_{1}, a_{2}, a_{j}\right)$. We deduce that $a_{5} \in \Lambda\left(a_{2}, a_{j}\right), \quad a_{6} \in \Lambda\left(a_{1}, a_{6}\right)$ and that $a_{k} \in \mathbb{Q}\left\{a_{1}^{2}, a_{2}^{2}, a_{1} a_{j}, a_{2} a_{j}\right\}$ where $k=\{3,4\}-j$.

1) If $a_{k} \in \mathbb{Q} a_{1}^{2}$, then $a_{6}=a_{1} a_{j}$ and $a_{5} \in \mathbb{Q}\left\{a_{2}^{2}, a_{2} a_{j}\right\}$. As $\left|a_{5}\right| \leq\left|a_{6}\right|$, then $a_{5} \in \mathbb{Q} a_{2}^{2}$, and so $\left|a_{i}\right|$ is even for all $i=1, \cdots, 6$, but this implies that $\chi_{c} \neq 0$.

2) If $a_{k} \in \mathbb{Q} a_{2}^{2}$, then necessary $\alpha_{1}^{2}=0$ and $\alpha_{j} \alpha_{k}=\lambda \alpha_{2}^{2} \alpha_{j}=\lambda^{\prime} \mu$. Hence $f d(X)=2\left|a_{2}\right|+\left|a_{j}\right|$ and $a_{5} \in \mathbb{Q} a_{2} a_{j}, a_{6} \in \mathbb{Q} a_{j}^{2}$. Since $\left|a_{j}\right|$ and $\left|a_{2}\right|$ are both even, then $f d(X)=\left|a_{1}\right|+\left|a_{6}\right|$ and $\left|a_{6}\right|=2\left|a_{j}\right|$. So $\left|a_{i}\right|$ is even for all $i=1, \cdots, 6$, but this leads to the contradiction $\chi_{c} \neq 0$.

3) If $a_{k} \in \mathbb{Q} a_{1} a_{j}$, then $\alpha_{1}^{2}=0$ and $\alpha_{1} \alpha_{j}^{2}=\lambda \mu$. Suppose that $\alpha_{2}^{2}=0$ and discuss two cases.

a) $a_{2}^{2} \in \mathbb{Q} a_{1} a_{j}$, then $\left|a_{1}\right|,\left|a_{2}\right|$ and $\left|a_{3}\right|$ are even and $\chi_{c} \neq 0$.

b) $a_{2}^{2} \in \mathbb{Q} a_{5}$, then $f d(X)=3\left|a_{2}\right|$ is even, but also $f d(X)=\left|a_{1}\right|+\left|a_{6}\right|=\left|a_{1}\right|+2\left|a_{j}\right|$, then $\left|a_{1}\right|$ is even. Therefore $\left|a_{i}\right|$ is even for all $i=1, \cdots, 6$ and $\chi_{c} \neq 0$.

4) If $a_{k} \in \mathbb{Q} a_{1} a_{j}$, then $\alpha_{1}^{2}=\alpha_{2}^{2}=0$ and $\alpha_{j} \alpha_{k}=\lambda \alpha_{2} \alpha_{j}^{2}=\lambda^{\prime} \mu$ (Poincar duality). Hence $a_{5} \in \mathbb{Q} a_{j}^{2}$, $a_{6} \in \mathbb{Q} a_{j}^{3}$ and $f d(X)=\left|a_{2}\right|+2\left|a_{j}\right|=\left|a_{1}\right|+3\left|a_{j}\right|$. So $\left|a_{1}\right|+\left|a_{j}\right|=\left|a_{2}\right|<\left|a_{j}\right|$.

Proposition 23 If $\left|\alpha_{1}\right|<\left|\alpha_{2}\right|<\left|\alpha_{3}\right|<\left|\alpha_{4}\right|<\left|\alpha_{5}\right|<\left|\alpha_{6}\right|$ and if $\left|\left\{a_{1}, a_{2}, a_{3}, a_{4}\right\} \cap V\right|=3$, then $X$ have one of the following r.h.t:

- $E$ : the total space of the fiber bundle with $\mathbb{S}^{2 p+1} \times$ 
$\mathbb{S}^{2 q+1}$ as base space,

- $\mathbb{S}^{n_{1}} \times \mathbb{S}^{n_{2}} \times \mathbb{S}^{n_{3}}$ where $n_{1}, n_{2}$ and $n_{3}$ are both even.

Proof. We know by the precedent lemmas and by the Poincar duality that $\alpha_{k} \alpha_{j}=\alpha_{1} \alpha_{2} \alpha_{j}=\mu$. Put $a_{5}=a_{1} a_{k}$ and $a_{6}=a_{2} a_{j}$, then $\alpha_{1}^{2}=0$ and $\alpha_{2}^{2}=\lambda \alpha_{1} \alpha_{j}$. We distinguish two cases:

1) $\lambda \neq 0$, then $\left|a_{1}\right|$ and $\left|a_{j}\right|$ are both odd because that $\chi_{c} \neq 0$. Replace $\lambda a_{1}$ by $a_{1}$ and put $\lambda=1$, then the minimal model of $X$ is of the form

$\left(\Lambda\left(x, y_{1}, y_{2}, y\right), d\right)$ with $\left|y_{1}\right|=2 p+1<|x|=2 n<\left|y_{2}\right|=$ $2 q+1$ and $d y=x^{2}-y_{1} y_{2}$. Hence $X \sim E$ where $\mathbb{S}^{2 n} \rightarrow$ $E \rightarrow \mathbb{S}^{2 p+1} \times \mathbb{S}^{2 q+1}$ is a fibration of the KS-complex

$$
\begin{aligned}
\left(\Lambda\left(y_{1}, y_{2}\right), 0\right) & \rightarrow\left(\Lambda\left(y_{1}, y_{2}\right) \otimes \Lambda(x, y), d\right) \\
& \rightarrow(\Lambda(x, y), \bar{d}) .
\end{aligned}
$$

2) $\lambda=0$, then $X$ have the minimal model $\left(\Lambda\left(x, y_{1}, y_{2}, y\right), d\right)$ with $d y_{1}=d y_{2}=0$ and $d y=x^{2}$, i.e., $X \sim \mathbb{S}^{n_{1}} \times \mathbb{S}^{n_{2}} \times \mathbb{S}^{n_{3}}$ with $n_{1}, n_{2}$ and $n_{3}$ are both even.

\section{Acknowledgements}

The authors would like to think the anonymous reviewers for their constructive comments and suitable advices on an earlier draft of this paper. It is also a pleasure to thank Paul Goerss and Kathryn Hess for their interest and encouragement. The authors are grateful to Hiroo Shiga and Toshihiro Yamaguchi for the several email discussion exchanged before the submission of this paper.

\section{REFERENCES}

[1] G. Bazzoni and V. Muõz, "Rational Homotopy Type of Nilmanifolds Up to Dimension 6," arXiv: 1001.3860v1, 2010.

[2] J. B. Friedlander and S. Halperin, "An Arithmetic Characterization of the Rational Homotopy Groups of Certain Spaces," Inventiones Mathematicae, Vol. 53, No. 2, 1979, pp. 117-133. doi:10.1007/BF01390029

[3] Y. Felix, S. Halperin and J.-C. Thomas, "Rational Homotopy Theory," Graduate Texts in Mathematics, Vol. 205, Springer-Verlag, New York, 2001.

[4] P. Griffiths and J. Morgan, "Rational Homotopy Theory and Differential Forms," Progress in Mathematics, Birkhäuser, Basel, 1981.

[5] S. Halperin, "Finitness in the Minimal Models of Sullivan," Transactions of American Mathematical Society, Vol. 230, 1977, pp. 173-199.

[6] I. M. James, "Reduced Product Spaces," Annals of Mathematics, Vol. 62, No. 1, 1955, pp. 170-197. doi: $10.2307 / 2007107$

[7] G. M. L. Powell, "Elliptic Spaces with the Rational Homotopy Type of Spheres," Bulletin of the Belgian Mathematical Society-Simon Stevin, Vol. 4, No. 2, 1997, pp. 251-263.

[8] H. Shiga and T. Yamaguchi, "The Set of Rational Homotopy Types with Given Cohomology Algebra," Homology, Homotopy and Applications, Vol. 5, No. 1, 2003, pp. 423436. 